\title{
BMJ Open Group Pregnancy Care for refugee background women: a codesigned, multimethod evaluation protocol applying a community engagement framework and an interrupted time series design
}

Elisha Riggs (D) ,,2 Jane Yelland (1) , , Fiona K Mensah (D) ,,3 Lisa Gold (D) ,4,5 Josef Szwarc, ${ }^{6}$ Ida Kaplan, ${ }^{6}$ Rhonda Small (D) , 7,8 Philippa Middleton (D) , 9,10 Ann Krastev, ${ }^{1}$ Ellie McDonald (D) , ${ }^{1}$ Christine East (D) , ${ }^{11}$ Caroline Homer (D) , 12 Natalija Nesvadba, ${ }^{13}$ Laura Biggs (D) , Jeffrey Braithwaite (D) , ${ }^{14}$ Stephanie J Brown (D) 1,2,3,9

To cite: Riggs E, Yelland J, Mensah FK, et al. Group Pregnancy Care for refugee background women: a codesigned, multimethod evaluation protocol applying a community engagement framework and an interrupted time series design. BMJ Open 2021;11:e048271. doi:10.1136/ bmjopen-2020-048271

- Prepublication history for this paper is available online. To view these files, please visit the journal online (http://dx.doi. org/10.1136/bmjopen-2020048271).

Received 23 December 2020 Accepted 11 June 2021

Check for updates

(c) Author(s) (or their employer(s)) 2021. Re-use permitted under CC BY-NC. No commercial re-use. See rights and permissions. Published by BMJ.

For numbered affiliations see end of article.

Correspondence to A/Prof Elisha Riggs; Elisha.riggs@mcri.edu.au

\section{ABSTRACT}

Introduction Pregnancy and early parenthood are key opportunities for interaction with health services and connecting to other families at the same life stage. Public antenatal care should be accessible to all, however barriers persist for families from refugee communities to access, navigate and optimise healthcare during pregnancy. Group Pregnancy Care is an innovative model of care codesigned with a community from a refugee background and other key stakeholders in Melbourne, Australia. Group Pregnancy Care aims to provide a culturally safe and supportive environment for women to participate in antenatal care in a language they understand, to improve health literacy and promote social connections and inclusion. This paper outlines Froup Pregnancy Care and provides details of the evaluation framework.

Methods and analysis The evaluation uses community-based participatory research methods to engage stakeholders in codesign of evaluation methods. The study is being conducted across multiple sites and involves multiple phases, use of quantitative and qualitative methods, and an interrupted time series design. Process and cost-effectiveness measures will be incorporated into quality improvement cycles. Evaluation measures will be developed using codesign and participatory principles informed by community and stakeholder engagement and will be piloted prior to implementation.

Ethics and dissemination Ethics approvals have been provided by all six relevant authorities. Study findings will be shared with communities and stakeholders via agreed pathways including community forums, partnership meetings, conferences, policy and practice briefs and journal articles. Dissemination activities will be developed using codesign and participatory principles.

\section{Strengths and limitations of this study}

- The conceptual framework for Group Pregnancy Care is informed by recognition of the diverse experiences of families of refugee background and the need for trauma informed approaches in health and social care and research.

- The evaluation involves a comprehensive, multimethod evaluation framework including interrupted time series, process measures and cost-effectiveness analysis.

- Partnerships and community engagement have informed the codesign of the evaluation methods.

- Capacity building and employment of refugee background staff is central to conduct the evaluation and to support women's participation.

- Staffing and resource constraints coupled with the COVID-19 pandemic have limited the capacity of health services to implement the intervention. Pandemic social distancing measures also limited the capacity to implement some elements of the evaluation.

\section{INTRODUCTION}

The United Nations estimates there are approximately 70 million forcibly displaced people globally. ${ }^{1}$ Each year, the Australian Government sets the number of visas that may be granted to those in humanitarian need. This quota has been around 13750 places annually, with some variation. In 2017, an additional 12000 places were provided for people displaced from Syria and Iraq. In the state of Victoria, Australia, $40 \%$ of all women giving birth in 2017 were born overseas, with the majority of migrant 
women coming from a country where English is not the main language. ${ }^{2}$ This equates to over 31000 women of non-English speaking background giving birth in Victoria each year. In Victoria's largest metropolitan maternity hospitals around $10 \%$ of all women giving birth are of refugee background. ${ }^{3}$

Women from refugee backgrounds have high rates of stillbirth and perinatal mortality. ${ }^{45}$ They also have a high risk of physical, mental and social health problems related to experiences of hardship, stress and experience of persistent disadvantage in the high-income countries in which they settle. ${ }^{6-8}$ The loss of family members through death, detention or separation is common, with pervasive and long-term consequences for mental health, family functioning and social cohesion. ${ }^{910}$

There is accumulating evidence that exposure to stress and trauma preceding, during and after pregnancy contributes to a range of adverse outcomes (eg, infants born preterm, small for gestational age or with low birth weight), with the potential to affect health across the life course. ${ }^{1-18}$ Further, the psychological and social impacts of torture and other traumatic events can often be experienced intergenerationally. ${ }^{101215}$

The provision of effective high-quality care during pregnancy is critical for healthy mothers and babies. ${ }^{19}$ Pregnancy care should provide an opportunity to identify and attend to potentially modifiable social risk factors (eg, social isolation, stress, trauma, low health literacy, family violence and smoking).$^{20-25}$ However, the evidence regarding the effectiveness of antenatal interventions addressing social risk factors is mixed. For example, Kiely et al found that a relatively brief psychosocial intervention in pregnancy resulted in a reduction of intimate partner violence (IPV) and improved pregnancy outcomes among African-American women. ${ }^{26}$ In contrast, a systematic review pooling evidence from nine randomised controlled trials (RCTs) evaluating pregnancy interventions for IPV concluded that stronger, high-quality research evidence is needed to clarify which interventions should be adopted. ${ }^{27}$

Families of refugee background encounter significant barriers accessing and using public maternity services and early maternal and child health (MCH) services. ${ }^{28} 29$ Within these systems of care, failure to identify and address clinical and social risks for poor maternal and infant outcomes places mothers and unborn babies at significant risk of adverse outcomes. ${ }^{19}{ }^{30}$ Supporting women to develop health literacy by tailoring antenatal and postnatal care to address their specific needs for information and build social connections for support has been shown to improve both healthcare access and engagement, and to enhance women's ability to make health decisions for themselves and their children. ${ }^{31-33}$

In this paper, we outline the evaluation framework for an innovative model of Group Pregnancy Care
(GPC) for women of refugee background that is currently being implemented in Melbourne, Australia. GPC aims to provide a culturally safe and supportive environment for women to actively participate in their healthcare ${ }^{34}$ GPC aims to improve MCH outcomes by increasing engagement with antenatal care, providing early postnatal care, overcoming language and health literacy barriers and decreasing social isolation.

\section{GPC FOR WOMEN AND THEIR FAMILIES FROM A REFUGEE BACKGROUND}

WHO identified GPC as having the potential to meet the complex needs of populations vulnerable to poor outcomes, ${ }^{19}$ with the Australian antenatal care guidelines outlining the potential benefits to women from refugee backgrounds. ${ }^{35}$ Group-based pregnancy models typically involve a midwife providing antenatal care and education to a number of women at the same time. The group setting provides a forum for sharing information and developing supportive social networks. The premise of the model is that women learn best from each other's experience, with facilitated discussion focusing on what women want to know.

The evidence in this field is building, with studies indicating improvements in preterm birth and low birth weight, ${ }^{36}$ maternal knowledge and patient satisfaction, ${ }^{37-39}$ social support ${ }^{40}$ and reduced costs of healthcare provision. ${ }^{41}$ However, a Cochrane systematic review including four randomised and quasi-RCTs ( $\mathrm{n}=2350$, English speaking women) evaluating group antenatal care found no clear evidence of improvement in rates of preterm birth, low birth weight, small-for-gestational age infants or perinatal mortality comparing group-based models of antenatal care with one to one antenatal care. ${ }^{42}$ The authors concluded that the number of women included in the review was too small to provide adequate power for meaningful comparisons, and further research was needed. Since this review, there has been a rapid emergence of new evidence supporting group-based models of pregnancy care. A systematic overview of group prenatal care in high-risk populations identified a range of improved outcomes for women identified as having a 'high-risk' pregnancy. ${ }^{43}$ This included a $33 \%-41 \%$ decrease in preterm birth for low income and African American women, and increased antenatal care attendance for women with opioid addiction, adolescents and lowincome groups observed in a number of studies in the review. The purpose of this review was to summarise the state of the evidence, yet pooled analyses were not attempted. Other American studies involving African American and Medicaid (public health insurance in the USA) eligible women have identified a reduction in low birth weight, caesarean birth, low 5 min Apgar scores and neonatal intensive care unit admissions for women who attended the group model compared with 
standard care. ${ }^{44-46}$ However, none of these studies have specifically focused on women from refugee backgrounds.

\section{CODESIGN AND IMPLEMENTATION OF A NEW MODEL OF GPC}

All Australian residents have access to free pregnancy care at public hospitals, and from public MCH (and other community health) services, and subsidised care from community-based general practitioners through Australia's universal public health insurance scheme (Medicare). ${ }^{47}$ Three quarters of women in Australia give birth in a public hospital and around a quarter of women give birth in a private hospital. ${ }^{48}$

The Bridging the Gap Partnership (2014-2016) was a consortium comprising 13 agencies, who came together with a shared goal of reducing disparities in $\mathrm{MCH}$ outcomes among families of refugee background living in Melbourne's south eastern and western suburbs. The Partnership implemented a series of quality improvement initiatives over a 4-year period. ${ }^{39}$ One of these initiatives involved the co-design and piloting of GPC for Karen families (from Burma) living in an outer western region of Melbourne, Australia. ${ }^{34}$ This was the first programme of its kind in Australia, involving inter-agency collaboration between public maternity hospitals, refugee support services and publicly funded MCH services. The pilot was evaluated in 2016 and details of the codesign process and qualitative evaluation have been published elsewhere. ${ }^{34}$

The key elements of GPC are outlined in box 1 and described on the study website.$^{50}$ In brief, the model involves multifaceted, culturally appropriate, preventive and health promoting healthcare, information and support for women of refugee background during and after pregnancy in a community setting. The model of care is delivered by a multidisciplinary team of five staff. Fortnightly group information sessions are co-facilitated by a midwife, MCH nurse and bicultural worker. Clinical antenatal care is provided by a second midwife and an on-site interpreter alongside the group sessions. GPC is cost-free for clients; provides pregnancy care and information that is woman-directed, culturally appropriate and in women's language; and facilitates referrals, for example, social work, housing services. Women can enrol to attend the fortnightly group information sessions at any stage of pregnancy.

Figure 1 outlines the programme logic for GPC. GPC is underpinned by the Trauma Recovery Framework developed by the Victorian Foundation for Survivors of Torture (Foundation House).$^{51}$ The family context and promoting positive outcomes for the whole family are central to GPC. A fundamental premise of GPC is that by creating culturally safe places for women to connect, access information and strengthen health literacy and self-efficacy, the key elements of GPC will contribute to improved birth and family health outcomes. We
Box 1 Key elements of Group Pregnancy Care (GPC) for refugee background women

Key elements:

- Local partnerships between public maternity hospitals, maternal and child health services and multicultural agencies. Partnership meetings are held quarterly and both managers and staff are invited to attend.

- Community and stakeholder engagement in the codesign of each new local programme. GPC is tailored to meet the needs of a specific cultural group.

- Establishment of a multidisciplinary team including two midwives, a maternal and child health nurse, a professional interpreter and a community and language-specific bicultural worker; with the same team delivering GPC each session, with designated back-up staff available if needed.

- Women are invited to participate by general practice referral, hospital booking or through community networks.

- Women-directed group information sessions with women from early to late pregnancy, cofacilitated by a midwife, maternal and child health nurse and a bicultural worker.

- Pregnancy care (as per standard hospital schedule of visits) with a midwife and professional interpreter held at the same time as the group information session.

- Home visits by the same maternal and child health nurse and bicultural worker up to 4 months post partum (if needed).

- Locating GPC in a community setting close to where families live (eg, maternal and child health centre).

Flexibility to embed the model in ways that work for communities and health services.

expect that GPC should be able to change individual behaviours (eg, self-efficacy, health literacy). As outlined in figure 1 , these determinants are on the pathway to improved birth and family health outcomes.

We have defined some key terms here:

\section{Self-efficacy}

Refers to an individual's confidence in their ability to complete a task or achieve a goal or be able to cope with a given situation based on the skills they have and the circumstances they face.

\section{Health literacy}

Is about how people access health and healthcare information, understand the information and how they apply that information to their lives by making decisions and acting on it.

\section{Cultural safety}

Seeks to achieve better care through being aware of cultural difference, giving consideration of power relationships, implementing reflective practice, and makes systems and organisations responsible to ensure that service environments are safe for everyone-regardless of their expressed or assumed culture.

Nineteen women participated in a qualitative evaluation of the GPC pilot. Results showed that GPC provides a space for women to feel like they belong, connect with their community networks and find a sense of kinship, 


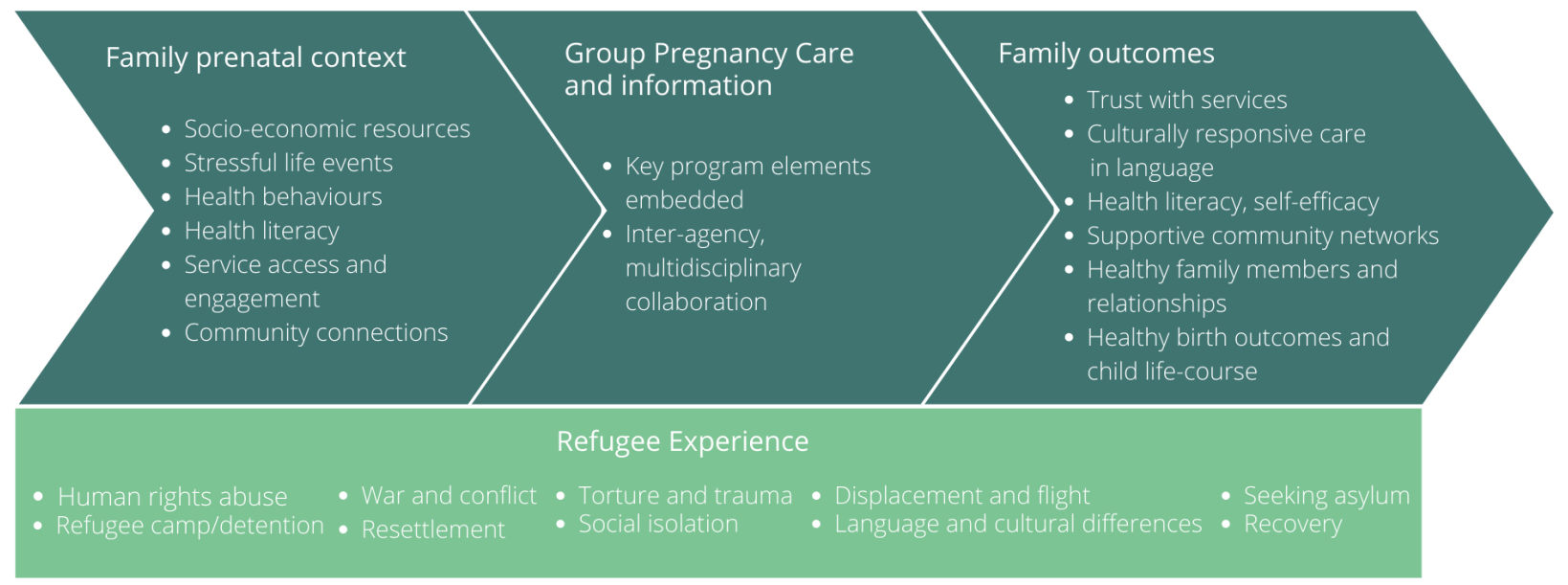

Figure 1 Conceptual model of Group Pregnancy Care to improve outcomes.

family and community in Melbourne. ${ }^{34}$ A key finding was the pivotal role of the bicultural worker in leveraging her own community networks for women to find out about GPC, and enabling trust and understanding between healthcare providers. The bicultural worker also played a valuable role building the knowledge and skills of other team members in culturally appropriate and sensitive ways of working with women and families of refugee background. ${ }^{46}$ Based on the positive findings of this preliminary evaluation, the Victorian Government supported scale-up and a robust evaluation across several sites.

\section{SCALING UP GPC}

\section{Partnership and governance}

The Murdoch Children's Research Institute (MCRI) is the lead agency and oversees the GPC study in partnership with Foundation House. Foundation House is the major statewide provider of counselling and advocacy services for people of refugee background. Building on community, research and health service partnerships established by the Bridging the Gap Partnership, the Lead Investigator brought together a team of study investigators with expertise in refugee health, community engagement, perinatal mental health, midwifery, implementation science, biostatistics and health economics to oversee the evaluation. The original partnership expanded to include eight health services; two refugee agencies; and three Victorian government department and peak governmental bodies. In addition, Local Partnership Groups have been formed comprising key managers and staff from the services involved to oversee site-specific codesign and implementation.

\section{Partnerships for sustainability}

The context for implementing and evaluating GPC is complex. Each GPC site involves a public hospital with competing demands for acute care resources. The implementation of GPC requires substantial investment from all stakeholders into the partnership relationship. All aspects of design, system readiness and workforce development have been developed within the existing resources of each partner agency. The partner agencies have provided a substantial investment of time, energy and enthusiasm for trying out GPC to improve care and outcomes for refugee families. This approach was adopted as a strategy to support sustainability of GPC within health services.

Pressures on the health agencies taking part include rising birth rates and changing demographics (ie, population growth, new refugee populations) in the regions served by participating hospitals, coupled with organisational restructuring and fluctuations in workforce supply. In addition, national and state refugee and asylum seeker health service eligibility policies compound the challenges for services in meeting the needs of women and families newly arrived in Australia. Despite these systemic challenges, the investment in partnerships is anticipated to promote direct and sustained translation into practice. ${ }^{52}$

\section{GPC staffing}

GPC staff (eg, midwives, MCH nurses, bicultural workers, interpreters) have been identified by service managers or via internal Expression of Interest pathways. Bicultural workers were drawn from staff already employed by one of the partner agencies (Victorian Cooperative on Children's Services for Ethnic Groups, known as VICSEG New Futures). All GPC staff participated in tailored professional development provided by Foundation House ${ }^{53}$ and the Groupwork Centre. ${ }^{54}$ Training included traumainformed approaches to care, ${ }^{51}$ skills development in group cofacilitation, creating group safety, self-care and reflective practice.

\section{Communities participating in GPC}

The partner agencies committed to expanding GPC with four refugee background communities: Karen (from Burma), Afghan, Assyrian Chaldean (from Iraq and Syria) and Vietnamese communities. Priority for these populations was based on:

1. Evidence of poor maternal, perinatal and child health outcomes and under utilisation/lack of engagement with services (based on service data). 
2. $>100$ births per annum in the country/language group at partner hospitals.

3. Social risks within communities as identified by partner agencies (access issues, isolation, family violence, low health literacy, requirement for interpreters).

Drawing on learnings from the pilot study, women are invited to participate in GPC when they book in for pregnancy care at the participating hospital or they may be referred through community/social networks and other health and social care services (eg, GP clinics).

\section{STUDY AIMS}

The specific aims of the evaluation are to:

1. Evaluate the effectiveness of GPC in improving timely access and engagement with preventive healthcare.

2. (A) Identify attributes of GPC (ie, frequency of attendance at the group sessions, acceptability of GPC) that are associated with healthcare access and engagement and improved maternal and child outcomes and (B) monitor adverse maternal, perinatal and infant health outcomes of women participating in GPC.

3. Examine mothers' progression in health literacy, social and emotional well-being and experience of care associated with participation in GPC.

4. Estimate the potential cost-offsets from improved $\mathrm{MCH}$ outcomes relative to the costs of implementing GPC and cost-effectiveness.

We hypothesise that GPC will increase access and engagement with prenatal care (primary outcome for evaluation), and that participation in GPC will result in subsequent changes in individual health behaviours (eg, self-efficacy, health literacy) that are on the pathway to improved birth and maternal health outcomes.

\section{STUDY DESIGN AND EVALUATION FRAMEWORK}

The evaluation framework has been developed using community-based participatory research methods to engage stakeholders in codeign of the evaluation methods. The study is being conducted across multiple sites and involves multiple phases, use of quantitative and qualitative methods, including an interrupted time series design. The time series design uses routinely collected hospital data to compare health service use and maternal and infant outcomes preceding and over the period of implementation of GPC.

To explore GPC inputs and attributes, a prospective cohort of mothers participating in GPC across all sites is being recruited. Interviews are conducted at 30 weeks' gestation and at 4 months post partum. To further enhance understanding of scale-up and implementation, we are conducting focus groups with participating women and service providers implementing the model of care. Finally, an assessment of cost effectiveness of GPC is being undertaken (figure 2).

\section{Scaling up complex interventions}

Scaling 'up' and scaling 'out' is a challenging process and involves changing systems, institutions, policies, practices and the ethos of people, organisations and systems. ${ }^{55-57}$ Not all elements are in the control of those wanting to implement the initiative. To determine what enables the capacity of systems to scale-up innovation (or not) requires a flexible and multifaceted approach to evaluation. The GPC study incorporates methods designed to answer questions that arise during implementation, enabling timely feedback to support scalability and sustainability.

Our approach to scaling up draws on implementation science, complexity theory and social science-that is, scale-up as structured improvement. ${ }^{57}$ To study the ecological properties of GPC as a complex intervention $^{58}$ addressing socially determined health inequalities, multiple methods and data sources, including both qualitative and quantitative data are being used. Rather than demonstrating success of the scale-up of GPC by measuring fidelity of its replication alone, we seek to

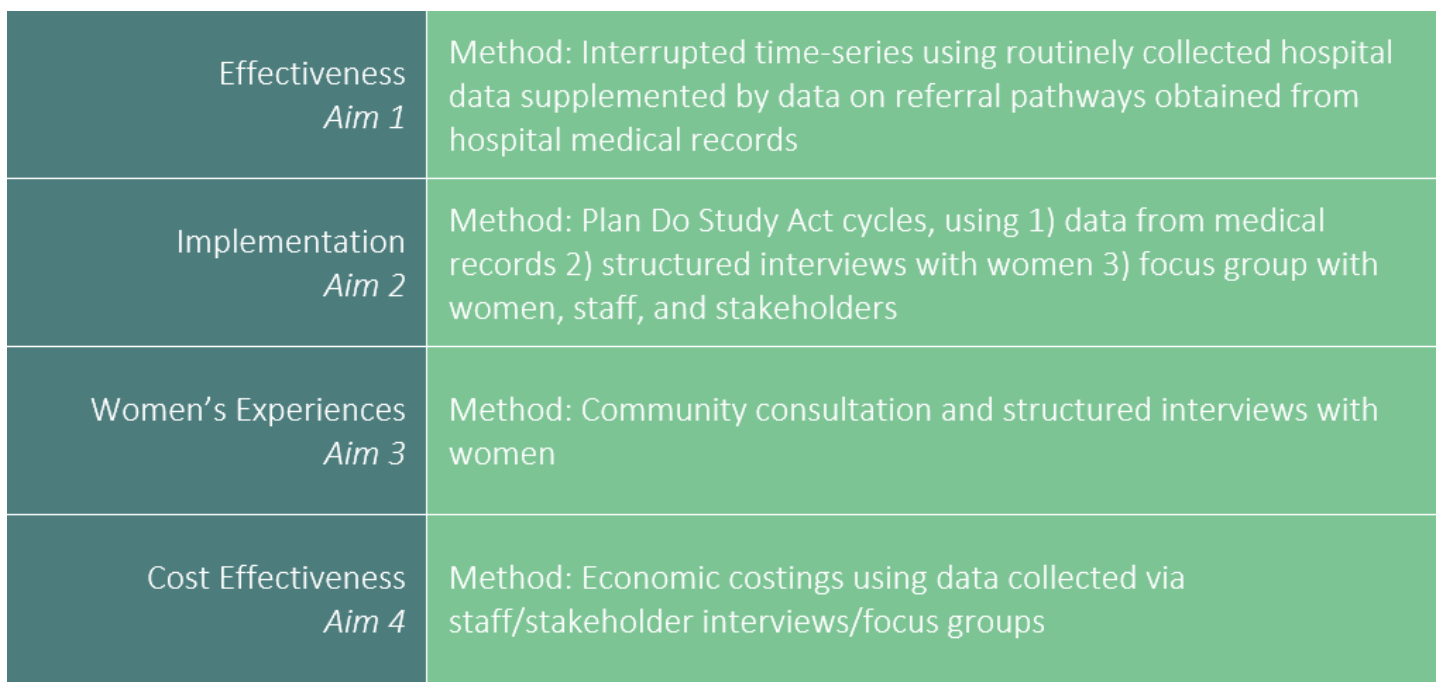

Figure 2 Evaluation overview. 
generate a nuanced understanding of what changed during implementation, why and how. We will draw on: (1) implementation science which takes a structured approach to developing, replicating and evaluating interventions in multiple sites; (2) complexity science which encourages a flexible and adaptive approach to change in dynamic systems and (3) social science which aims to consider why people act in the way they do, encompassing the organisational and wider social forces that shape and constrain people's actions. ${ }^{57}$ These approaches will be used in combination to understand the challenges of spread and scale-up of a complex intervention. Our model of GPC as a complex intervention has been codesigned with each community, as we know that culturally adapting interventions can increase salience, acceptability and uptake. ${ }^{59}$ Currently, there is insufficient evidence on the clinical effectiveness or cost-effectiveness of such an approach. We hope to contribute to this evidence base with learnings that can be translated to other settings, and more broadly, to policy and practice guidance. ${ }^{60}$

\section{METHODS AND ANALYSIS}

\section{Patient and public (community) involvement}

The priorities of the partner agencies and the experiences of the women participating in the pilot study have been critical to the design of this study, in particular the research questions, outcome measures and translation strategies. Study findings will be shared via bicultural researchers at Community Advisory Groups and presentations at community forums. The following sections details the community involvement in the conduct of all aspects of the evaluation.

Partnerships, community engagement and capacity building underpin this study. For the past 9 years MCRI and Foundation House have been working in partnership to develop and implement a programme of collaborative, community-based, participatory research with refugee families focusing on social health and well-being of the whole family. Our commitment to respect, reciprocity and capacity building are fundamental to the way in which this study has been designed. Community engagement is a key strategy for ensuring that services are responsive to the needs of the communities which they serve.

The employment of linguistically and culturally matched bicultural research staff and provision of mentoring and training to build research capacity are central to this study. Drawing on the bicultural researchers' cultural knowledge, language skills and community networks is critical for establishing cultural safety. ${ }^{61}$ These participatory strategies aim to alleviate the unequal relationships between researchers and research participants that characterise traditional research approaches. ${ }^{62}$

\section{Role of bicultural researchers}

In addition to the four bicultural staff appointed to work with GPC implementation teams, four bicultural researchers have been employed as part of the MCRI evaluation team. The bicultural researchers speak the language of the communities participating in GPC and have extensive community knowledge and networks to support consultation and other research activities. Specific training for the bicultural researchers has included: skills building in research activities (recruitment, informed consent, data collection, data security); processes for supporting participants experiencing distress; and opportunities to practice and receive feedback with research team members on facilitating discussion groups and conducting interviews.

\section{Community advisory groups}

Following principles of cultural safety, ${ }^{61}$ Community Advisory Groups (CAGs) have been established for each refugee community. Our previous research with refugee background communities has established effective community engagement methodologies involving the employment of bicultural staff and the establishment of CAGs. ${ }^{63}$ Our methodology is inclusive, flexible and aims to build capacity and support ongoing community participation.

Recruitment of Community Advisors was undertaken by the bicultural researchers, with support from partner agencies. The aim of the CAGs is to involve women (and men where appropriate) from refugee backgrounds with a range of experiences (eg, new parents, Elders, religious leaders). Community consultation was conducted by the bicultural community researchers to identity appropriate community advisors. The CAGs meet at key points relevant to the evaluation. The role of community advisors has been to: (1) provide community perspectives to ensure the evaluation methods are appropriate and meaningful; (2) contribute to problem solving, interpretation and dissemination of the evaluation findings; (3) facilitate wider community engagement; and (4) provide a conduit between the MCRI research team and the community.

\section{Setting}

We planned to evaluate seven GPC programmes at four sites (in Melbourne Australia) involving six different refugee background communities (see figure 3 ). The sites include public maternity hospitals and MCH services in Melbourne's west, north and south east suburbs, all areas of high cultural diversity and increasing refugee settlement.

\section{Effectiveness (aim 1)}

Data extraction from the birthing outcomes system

To facilitate the interrupted time series analysis, all partner hospitals are extracting data from the electronic birthing outcomes system (BOS) for all women giving birth at each site for a 12-month period prior to commencement of GPC (baseline) and at 6 monthly intervals from implementation of GPC until completion. The BOS is a database collecting routine data including demographic information, service contact, screening results and maternal and neonatal outcomes (box 2). 

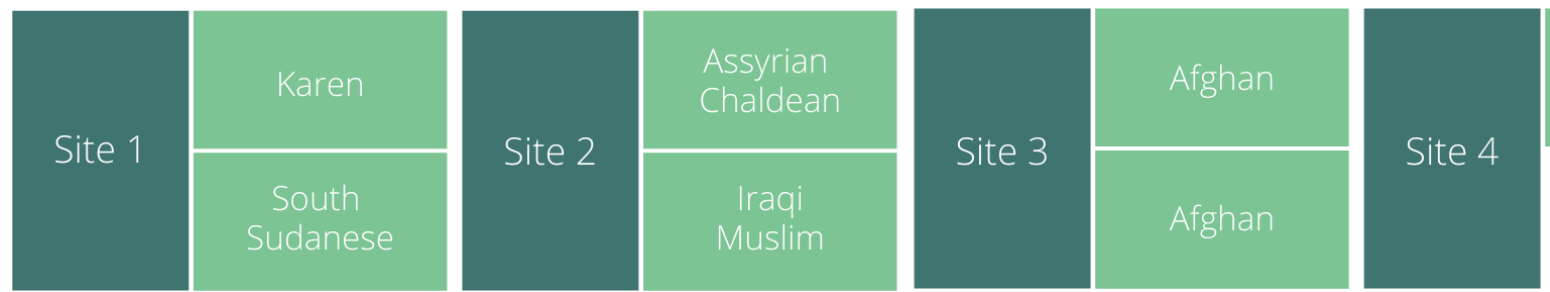

Vietnamese

Figure 3 Planned sites for Group Pregnancy Care.

We will categorise women according to whether they are Australian born or born overseas in an English-speaking country or non-English-speaking country. In addition, we will identify all women from the cultural backgrounds of women participating in GPC, identified by county of birth and language spoken. Women enrolled in GPC will be identified in the BOS by a code in the data set.

\section{Medical record data abstraction}

Non-routinely collected items, such as referral pathways,will be collected from the hospital records of women participating in GPC. Information will be abstracted on screening, referral and follow-up of medical and psychosocial issues, including: high blood pressure, gestational diabetes, suspected intrauterine growth restriction, maternal mental health and family violence. Data collection will be undertaken by a research midwife following a protocol using a standardised form. Deidentified data

\section{Box 2 Data to be extracted from hospital birthing}

\section{outcomes system}

\section{Primary outcome}

- Number of women attending $\geq 7$ antenatal clinic visits.

\section{Secondary outcomes}

- Pregnancy care: First antenatal visit $<14$ weeks, ultrasound scan $<14$ weeks, number of visits with a professional interpreter involved, screening/diagnostic tests for gestational diabetes at $<30$ weeks.

- Maternal pregnancy medical conditions and complications: hypertension, anaemia, pre-eclampsia, antepartum haemorrhage, gestational diabetes, threatened preterm labour, emergency department attendance.

- Pregnancy and birth events: induction of labour, method of birth, third or fourth degree tear.

- Maternal morbidity and mortality: intrapartum or postpartum haemorrhage, wound infection, admissions to intensive care, postdischarge readmission, maternal death.

- Infant outcomes: preterm birth ( $<37$ weeks), low birth weight $(<2500 \mathrm{~g})$, small/large for gestational age ( $<10$ th/90th centile), admission to neonatal/special care nursery for $>12$ hours, unplanned home birth, birth on way to hospital, stillbirth, neonatal death.

\section{Covariates}

\section{- Demographics}

Maternal country of birth, year of arrival in Australia, interpreter required, language(s) spoken, place of residence, maternal age at time of birth (years), relationship status.

Reproductive history: parity; plurality; gravidity, pre-existing medical conditions. will be entered into the Research Electronic Data Capture (REDCap) online software. ${ }^{64}$

\section{Comparative study populations, sample size and study power}

Based on attendance at the GPC pilot, we anticipated that 4 sites would implement 7 GPC programmes, providing an average of 74 women per group to participate in the evaluation. Allowing for an average loss of 4 women per group (5\%), our projected comparison is based on a total sample size of 490 women participating over the period of provision of GPC. Comparisons will be made with (1) 490 women receiving antenatal care in the 12 months preceding introduction of GPC and (2) 490 women contemporaneously enrolled in other models of care. These comparative populations will include women from the same cultural/language background receiving antenatal care at the same hospitals, selecting the most closely matched woman in regards to age and parity for each woman participating in GPC.

As the level of intragroup correlation in attendance is unknown but expected to be low, we will include an intraclass correlation in attendance by hospital for women not participating in GPC, ICC 0.001 and an ICC of 0.005 within groups for women participating in GPC. A level of $5 \%$ is considered significant for all comparisons. Using the hospital data as the basis for estimates, the comparisons would each provide $94 \%$ power to detect a minimum absolute difference in attendance at seven or more antenatal visits of $10 \%$ (from the current $76 \%$ to $86 \%$ ). Seven visits are considered the minimum for a healthy pregnancy without complications. ${ }^{35}$

\section{Statistical analysis}

(1) All statistical analyses will be performed using STATA V.15. ${ }^{65}$ An interrupted time-series comparison ${ }^{66}{ }^{67}$ will be used to investigate the difference in the primary outcome-proportion of mothers attending the recommended number of antenatal visits-associated with the introduction of GPC. This comparison will be made using multilevel regression models accounting for clustering of mothers within hospitals/pregnancy groups, autocorrelation of the observed primary outcome over time and potential period effects (eg, changes in the percentage of families of refugee background in the hospital service area). Models will thus include whether the timing of pregnancy was pre or post introduction of the GPC; and an additional item reflecting trend in antenatal attendance over the course of the evaluation to account for 
any contemporaneous patterns of attendance. Maternal characteristics specified a priori will be controlled for including: age; reproductive history and pregnancy complications (eg, parity, prior preterm birth, prior stillbirth); gestation at GPC enrolment; country of birth; year of arrival; and other sociodemographic characteristicsthis accounting for changes in the eligible population over the study period. (2) Comparisons between women contemporaneously participating in GPC and other models of care will be conducted using congruent modelling strategies replacing the comparator of whether the timing of pregnancy was pre or post introduction of GPC with the comparator of participating in GPC versus other models of care.

\section{Implementation (aim 2)}

We will use an approach to iterative, continuous quality improvement cycles called the Plan-Do-Study-Act (PDSA) method. ${ }^{68}$ This will involve collecting and analysing data and feeding it back to the Local Partnership Groups to refine GPC and continue to improve it. ${ }^{69}$ This feedback aims to support and strengthen potential for intervention sustainability. The partnership adopted the PDSA framework as a pragmatic method for implementing and testing changes through small rapid cycles of improvement, with flexibility to adapt change according to feedback and engage GPC staff/managers in each PDSA cycle. The PDSA method aims to provide a supportive process to trial new ideas, allowing for small failures without compromising overall standards of care.

Specifically, we will use the PDSA method to: (1) conduct an initial assessment of the key elements of the model from the perspective of women/families and staff taking part; (2) identify barriers and enablers for implementation, and (3) refine GPC elements and implement strategies to minimise barriers and maximise opportunities to achieve objectives. The researchers have used this method successfully in other maternity initiatives. ${ }^{70}$ Three data sources will be used to gather data.

1. Data will be abstracted from hospital records of women participating in GPC to inform improvement (as described above in Effectiveness).

2. Focus groups with women will be used to explore experiences of: GPC accessibility, content and relevance. A semistructured discussion guide will be developed in consultation with the bicultural researchers. A purposive sample of 5-8women from each GPC programme who have completed the 16-week postpartum interview will be recruited (four groups, $\mathrm{n}=20-30$ ). Invited women will have a variety of experiences related to: time in Australia, English language fluency and group attendance. Focus groups will be conducted in women's preferred language co-facilitated by the MCRI bicultural researchers and will be audio recorded (with informed consent), transcribed verbatim, translated to English and analysed using thematic analysis. ${ }^{71}$

3. Midwives, MCH nurses, interpreters, bicultural workers and management involved in delivery of the new model ( $\mathrm{n}=35-45$ participants) will be invited to participate in focus groups/interviews. Discussions will explore process evaluation measures including staff experiences of GPC implementation, cross-sector collaboration, capacity building, skill development, multidisciplinary teamwork, organisational and systems change, and sustainability.

\section{Women's experiences (aim 3)}

All women enrolled in GPC will be invited to complete two interviews with a bicultural researcher at approximately 28-32 weeks' gestation and again 3-4 months post partum. The MCRI bicultural researchers will recruit women and conduct the interviews.

\section{Design and translation of structured interview}

Standardised measures have been used where possible, and pre-tested to ensure cultural acceptability. All study materials were translated into required languages by a professional agency and with the assistance of the bicultural researchers, translated back into English to ensure high quality and accurate translations. ${ }^{72}$ Maternal interviews will be audio recorded (with participant consent) and transcribed into English by bicultural staff.

Women's mental health will be measured using the Hopkins Symptom Checklist and Harvard Trauma Questionnaire, developed for refugee populations. ${ }^{73}$ Working with the MCRI bicultural researchers and the CAGs, we pilot tested the Edinburgh Postnatal Depression Scale S $^{745}$ and the Composite Abuse Scale ${ }^{76}$ (measures experiences of physical and psychological abuse within intimate partner relationships) to determine acceptability, or not, by all communities participating in GPC. Other domains include: general demographics, health literacy, social connections and experiences of GPC.

\section{Recruitment of prospective pregnancy cohort}

The bicultural researchers will attend the GPC site corresponding to their community and explain their role in the evaluation. Eligible women are enrolled in GPC and $\geq 18$ years old. Women are not eligible to take part if they are too unwell to participate, have an intellectual disability or medical condition precluding them from giving informed consent (eg, psychotic illness).

Participant details will be stored on a REDCap database which produces a report based on women's estimated due date to notify the research team when a woman is due to be contacted to schedule the postnatal interview.

\section{Data analysis}

After the completion of each interview, the bicultural researchers will transcribe the audio-recording to provide a comprehensive interview transcript in English. All data collected in the interview will be manually entered by research team staff into REDCap. Quantitative data will be exported to Stata V.15 for scoring and analysis. Qualitative data will be exported to and managed in NVivo ${ }^{77}$ for thematic analysis. Four steps for thematic data analysis 
will be followed: immersion, coding, categorising and developing themes. ${ }^{71}$

\section{Cost effectiveness (aim 4)}

Data on resources used to deliver the intervention will be collected, including time commitments of paid staff and participating women. These will be valued $(\$ A)$ at standard unit costs (eg, salary scales, interpreter costs, travel) to calculate intervention costs specific to each site. Intervention costs will be combined with potential cost-offsets and outcomes data (box 2) in an economic evaluation that compares additional costs associated with GPC to changes in health outcomes (cost-consequences analysis) ${ }^{78}$ Potential cost offsets from improved MCH outcomes will be estimated by partner hospitals based on routine perinatal data and hospital financial systems data on in-hospital care costs. As part of the qualitative data collection with GPC staff and stakeholders, information will be collected to inform the economic evaluation.

\section{PROGRESS TO DATE}

\section{Implementation of GPC}

Planning for expansion of GPC to three new sites commenced in 2017. However, due to staffing and resource constraints at two of these sites, only the initial GPC pilot and one new GPC programme remain part of the evaluation (involving the Karen and Assyrian Chaldean communities). Additional funding was secured in late 2019 to conduct consultation with two additional communities (Sudanese/South Sudanese and Iraqi/Syrian Muslim) with a view to establishing two new GPC programmes to join the overall evaluation.

\section{Implications of COVID-19 pandemic}

In response to the global COVID-19 pandemic, Australia initiated strict internal lockdown policies to reduce the risk of community transmission in late March 2020. As a result, the two established GPC programmes paused provision of group sessions, and planning for the two new GPC programmes was put on hold. The two established programmes transitioned to virtual platforms for clinical and group-based information sessions from March 2020. Group sessions using an online platform were initiated in response to the ongoing need for women and families to connect to services and peers for information and support.

The evaluation has also continued, adapting to telephone/video interviews with women and staff/stakeholders. The abrupt disruption to GPC services flowing from the COVID-19 pandemic mean the numbers of women participating in GPC and available for the interviews will be lower than planned.

Given these circumstances, the intended sample size will not be achieved within the funded study period. At this point, interim analyses will be conducted and preliminary findings will be shared with the study partners and funders. Interim analyses will provide substantial outputs in regard to process and implementation learnings (aim 2) and participant experiences (aim 3) as well as preliminary exploration of responses in the primary outcome (proportion of women attending $\geq 7$ antenatal clinic visits-aim 1). Extension of the study with the two additional communities at the new sites for which funding has been secured plus continuation of GPC at the existing sites where feasible, will provide the opportunity to extend evaluation to achieve the intended sample size for the full comparisons (aim 1) and economic evaluation (aim 4).

\section{ETHICS AND DISSEMINATION \\ Ethical considerations}

Human Research Ethics Committee (HREC) approvals have been provided by all six relevant authorities through the Australian National Mutual Acceptance scheme, where permitted. Ethical amendments were sought for each stage of the study following community and partner organisation consultation to finalise each stage. This staged approach enables piloting and reflection on the cultural safety ${ }^{61}$ of the research activities and flexibility to refine research processes to ensure appropriateness and meaningfulness to community members, bicultural researchers and partners. At the time of submitting this protocol, HREC approval had been granted for all stages of the study with a modification for data abstraction pending at one hospital.

In Australia, there are specific ethical guidelines for conducting research with Aboriginal and Torres Strait Islander Communities. ${ }^{79}$ However, an equivalent national approach to mandatory ethical research guidelines for the engagement of refugee background communities does not exist. We are mindful of the ethical issues to consider when conducting research with people of refugee background. ${ }^{80}{ }^{81}$ As the concepts of research and ethics may be unfamiliar to some participants, we acknowledge the possibility that participants may feel anxious about their involvement. Concerns may be provoked when issues such as privacy, trust and confidentiality, audiorecording of interviews, etc are not clear or comfortable for the participant. The bicultural researchers will clarify the voluntary nature of research participation and encourage participants to ask questions to alleviate any concerns, as conducted in our previous research. ${ }^{63}$ A study distress protocol developed in partnership with Foundation House and used in our previous research studies will guide the researchers in situations where participants become distressed, require support or disclose issues related to mental health, family violence or participant/child safety.

HREC approvals: The Royal Children's Hospital 37025, HREC/17/RCHM/66, MCRI SSA/17/RCHM/97, Monash Health 17-424X, HREC/16/MONH/65, SSA/17/MonH/362, Northern Health HREC/17/ RCHM/66, SSA/17/NH/104, Western Health HREC/17/ RCHM/66, SSA/AU/5/C@E0314, Mercy Health 2017017, Victorian Department of Health and Human Services HHSD/19/174035. 


\section{Dissemination}

We have developed a comprehensive knowledge translation and dissemination plan in line with our values of reciprocity and collaboration. Including: sharing study findings with communities in accessible ways (via bicultural researchers at CAGs), presentations at community forums, partnership meetings, conferences, policy and practice briefs and publication of journal articles. All outputs will be available on the study website.

\section{Author affiliations}

${ }^{1}$ Intergenerational Health, Murdoch Childrens Research Institute, Parkville, Victoria, Australia

${ }^{2}$ Department of General Practice, The University of Melbourne, Melbourne, Victoria, Australia

${ }^{3}$ Department of Paediatrics, The University of Melbourne, Melbourne, Victoria, Australia

${ }^{4}$ Deakin Health Economics, Deakin University, Burwood, Victoria, Australia

${ }^{5}$ Population Health, Murdoch Children's Research Institute, Melbourne, Victoria,

Australia

${ }^{6}$ Victorian Foundation for Survivors of Torture, Melbourne, Victoria, Australia

${ }^{7}$ Judith Lumely Centre, La Trobe University, Melbourne, Victoria, Australia

${ }^{8}$ Department of Women's and Children's Health, Division of Reproductive Health, Karolinska Institute, Stockholm, Sweden

${ }^{9}$ Women and Kids, South Australian Health and Medical Research Institute, Adelaide, South Australia, Australia

${ }^{10}$ Faculty of Medical and Health Sciences, The University of Adelaide, Adelaide,

South Australia, Australia

${ }^{11}$ School of Nursing and Midwifery/Mercy Hospital for Women, La Trobe University, Melbourne, Victoria, Australia

${ }^{12}$ Maternal, Child and Adolescent Health Program, Burnet Institute, Melbourne, Victoria, Australia

${ }^{13}$ Multicultural Services, Mercy Hospitals Victoria Ltd, Melbourne, Victoria, Australia

${ }^{14}$ Australian Institute of Health Innovation, Macquarie University, North Ryde, New South Wales, Australia

\section{Twitter Elisha Riggs @_elisha_riggs}

Acknowledgements We acknowledge and thank the many health and social care professionals, managers, administrators and policy personnel engaged in Group Pregnancy Care in a myriad of ways. In particular, we would like to thank May Alqas Alias, Shadow Toke, Shogoufa Hydari, Sheri Shwe, Lien Tran, the Community Advisory Group members, the women and families participating in the Group Pregnancy Care study, Professor Jeremy Oats, Professor Louise Howard, Ms Donna Chesters, Ms Heidi Zwick, Ms Colleen Turner, Ms Janet Elefsiniotis, Ms Sayanti Bhatta, Ms Karen Diacono, Ms Pauline Petschel, Ms Ann McNair, Ms Catherine Fletcher, Ms Adele Mollo, Ms Elizabeth Murdoch, Sandra Papadopoullos and other key staff from Mercy Health (Werribee Mercy Hospital), Northern Health, Monash Health, Western Health, Wyndham City Council, Hume City Council, Brimbank City Council, Foundation House, VICSEG New Futures, the Groupwork Centre, and personnel at the Victorian Government Department of Health and Human Services and the Municipal Association of Victoria.

Contributors ER is the study principal investigator and drafted this protocol based on the project proposal and other planning documents involving many people as outlined in the author contribution and acknowledgements. JY, FKM, LG, JS, IK, RS, $\mathrm{PM}, \mathrm{CE}, \mathrm{CH}, \mathrm{JB}$ and SJB are study investigators and NN is a key stakeholder from a partner agency who have contributed to the development of the project proposal and project planning. FKM conducted sample size estimate calculations for the interrupted time series design. AK, EM and LB are employed on the project and have contributed to development of planning documents, conceptual framework, ethics submissions which involve many of the components outlined in this protocol, and drafting the manuscript. All authors contributed to drafting and finalising the manuscript.

Funding This work is supported by the Victorian Government Department of Premier and Cabinet's Strategic Partnerships Metro-Capacity Building and Participation Programme, the North Western Melbourne Primary Health Network and the Murdoch Children's Research Institute Population Heath Theme. Research at the MCRI is supported by the Victorian Government's Operational Infrastructure Support
Programme. JY was supported by a National Health and Medical Research Council (NHMRC) of Australia Translating Research into Practice Fellowship (1150566). SJB was supported by an NHMRC Senior Research Fellowship (1103976). FKM was supported by an NHMRC Career Development Fellowship (1111160).

Competing interests None declared.

Patient and public involvement Patients and/or the public were involved in the design, or conduct, or reporting, or dissemination plans of this research. Refer to the Methods section for further details.

Patient consent for publication Not required.

Provenance and peer review Not commissioned; externally peer reviewed.

Open access This is an open access article distributed in accordance with the Creative Commons Attribution Non Commercial (CC BY-NC 4.0) license, which permits others to distribute, remix, adapt, build upon this work non-commercially, and license their derivative works on different terms, provided the original work is properly cited, appropriate credit is given, any changes made indicated, and the use is non-commercial. See: http://creativecommons.org/licenses/by-nc/4.0/.

\section{ORCID iDs}

Elisha Riggs http://orcid.org/0000-0003-0799-7467

Jane Yelland http://orcid.org/0000-0003-0377-5824

Fiona K Mensah http://orcid.org/0000-0002-6951-9949

Lisa Gold http://orcid.org/0000-0002-2733-900X

Rhonda Small http://orcid.org/0000-0003-0345-4676

Philippa Middleton http://orcid.org/0000-0002-8573-338X

Ellie McDonald http://orcid.org/0000-0003-2295-4641

Christine East http://orcid.org/0000-0002-1196-8426

Caroline Homer http://orcid.org/0000-0002-7454-3011

Laura Biggs http://orcid.org/0000-0002-8500-1584

Jeffrey Braithwaite http://orcid.org/0000-0003-0296-4957

Stephanie J Brown http://orcid.org/0000-0001-9812-0067

\section{REFERENCES}

1 UNHCR. Global trends in forced displacement in 2018. Geneva, Switzerland: United Nations High Commissioner for Refugees, 2019.

2 Consultative Council on Obstetric and Paediatric Mortality and Morbidity. Victoria's mothers, babies and children 2018. Melbourne, Victoria: Victorian Government, 2019.

3 Yelland J, Mensah F, Riggs E, et al. Evaluation of systems reform in public hospitals, Victoria, Australia, to improve access to antenatal care for women of refugee background: an interrupted time series design. PLoS Med 2020;17:e1003089.

4 Drysdale $\mathrm{H}$, Ranasinha $\mathrm{S}$, Kendall $\mathrm{A}$, et al. Ethnicity and the risk of late-pregnancy stillbirth. Med J Aust 2012;197:278-81.

5 Gibson-Helm ME, Teede HJ, Cheng I-H, et al. Maternal health and pregnancy outcomes comparing migrant women born in humanitarian and nonhumanitarian source countries: a retrospective, observational study. Birth 2015;42:116-24.

6 Porter M, Haslam N. Predisplacement and postdisplacement factors associated with mental health of refugees and internally displaced persons: a meta-analysis. JAMA 2005;294:602-12.

7 Chen W, Hall BJ, Ling L, et al. Pre-migration and post-migration factors associated with mental health in humanitarian migrants in Australia and the moderation effect of post-migration stressors: findings from the first wave data of the BNLA cohort study. Lancet Psychiatry 2017;4:218-29.

8 Beiser M. Resettling refugees and Safeguarding their mental health: lessons learned from the Canadian refugee resettlement project. Transcult Psychiatry 2009;46:539.

9 Fazel M, Reed RV, Panter-Brick C, et al. Mental health of displaced and refugee children resettled in high-income countries: risk and protective factors. Lancet 2012;379:266-82.

10 Wilmsen B. Family separation and the impacts on refugee settlement in Australia. The Australian Journal of Social Issues 2013:48:241.

11 Heslehurst N, Brown H, Pemu A, et al. Perinatal health outcomes and care among asylum seekers and refugees: a systematic review of systematic reviews. BMC Med 2018;16:89.

12 Sangalang CC, Vang C. Intergenerational trauma in refugee families: a systematic review. J Immigr Minor Health 2017;19:745-54.

13 Kokou-Kpolou CK, Moukouta CS, Masson J, et al. Correlates of grief-related disorders and mental health outcomes among adult refugees exposed to trauma and bereavement: a systematic review and future research directions. J Affect Disord 2020;267:171-84. 
14 Heard-Garris NJ, Cale M, Camaj L, et al. Transmitting trauma: a systematic review of vicarious racism and child health. Soc Sci Med 2018;199:230-40.

15 Steel Z, Chey T, Silove D, et al. Association of torture and other potentially traumatic events with mental health outcomes among populations exposed to mass conflict and displacement: a systematic review and meta-analysis. JAMA 2009;302:537-49.

16 Garfin DR, Thompson RR, Holman EA. Acute stress and subsequent health outcomes: a systematic review. J Psychosom Res 2018;112:107-13.

17 von Werthern M, Robjant K, Chui Z, et al. The impact of immigration detention on mental health: a systematic review. BMC Psychiatry 2018;18:382.

18 Kuh D, Ben-Shlomo Y. A life course approach to chronic disease epidemiology. 2nd ed. Oxford: Oxford University Press, 2004.

19 World Health Organization. WHO recommendations on antenatal care for a positive pregnancy experience. Switzerland: World Health Organization, 2016.

20 Kuzawa CW. Pregnancy as an intergenerational conduit of adversity: how nutritional and psychosocial stressors reflect different historical timescales of maternal experience. Current Opinion in Behavioral Sciences 2020;36:42-7.

21 McDonald S, Kehler H, Bayrampour H, et al. Risk and protective factors in early child development: results from the all our babies (AOB) pregnancy cohort. Res Dev Disabil 2016;58:20-30.

22 Olander EK, Smith DM, Darwin Z. Health behaviour and pregnancy: a time for change. J Reprod Infant Psychol 2018;36:1-3.

23 Vehmeijer FOL, Guxens M, Duijts L, et al. Maternal psychological distress during pregnancy and childhood health outcomes: a narrative review. J Dev Orig Health Dis 2019;10:274-85.

24 Giallo R, Gartland D, Seymour M, et al. Maternal childhood abuse and children's emotional-behavioral difficulties: intergenerational transmission via birth outcomes and psychosocial health. J Fam Psychol 2020;34:112-21.

25 Chamberlain C, O'Mara-Eves A, Porter J, et al. Psychosocial interventions for supporting women to stop smoking in pregnancy. Cochrane Database Syst Rev 2017;2:Cd001055.

26 Kiely M, El-Mohandes AAE, Gantz MG, et al. Understanding the association of biomedical, psychosocial and behavioral risks with adverse pregnancy outcomes among African-Americans in Washington, DC. Matern Child Health J 2011;15 Suppl 1:S85-95.

27 Van Parys A-S, Verhamme A, Temmerman M, et al. Intimate partner violence and pregnancy: a systematic review of interventions. PLoS One 2014;9:e85084-e84.

28 Yelland J, Riggs E, Wahidi S, et al. How do Australian maternity and early childhood health services identify and respond to the settlement experience and social context of refugee background families? BMC Pregnancy Childbirth 2014;14:348.

29 Riggs E, Davis E, Gibbs L, et al. Accessing maternal and child health services in Melbourne, Australia: reflections from refugee families and service providers. BMC Health Serv Res 2012;12:117.

30 Flenady V, Wojcieszek AM, Middleton P, et al. Stillbirths: recall to action in high-income countries. Lancet 2016;387:691-702.

31 Mobley SC, Thomas SD, Sutherland DE, et al. Maternal health literacy progression among rural perinatal women. Matern Child Health J 2014;18:1881-92.

32 Carroll LN, Smith SA, Thomson NR. Parents as teachers health literacy demonstration project: integrating an empowerment model of health literacy promotion into home-based parent education. Health Promot Pract 2015;16:282-90.

33 Small R, Taft AJ, Brown SJ. The power of social connection and support in improving health: lessons from social support interventions with childbearing women. BMC Public Health 2011;11 Suppl 5:S4.

34 Riggs E, Muyeen S, Brown S, et al. Cultural safety and belonging for refugee background women attending group pregnancy care: an Australian qualitative study. Birth 2017;44:145-52.

35 Department of Health. Clinical practice quidelines: pregnancy care. Canberra, Australia: Australian Government Department of Health, 2018.

36 Cunningham SD, Lewis JB, Shebl FM, et al. Group prenatal care reduces risk of preterm birth and low birth weight: a matched cohort study. J Womens Health 2019;28:17-22.

37 Picklesimer A, Heberlein E, Covington-Kolb S. Group prenatal care: has its time come? Clin Obstet Gynecol 2015;58:380-91.

38 Hetherington E, Tough S, McNeil D, et al. Vulnerable women's perceptions of individual versus group prenatal care: results of a cross-sectional survey. Matern Child Health J 2018;22:1632-8.

39 Liu R, Chao MT, Jostad-Laswell A, et al. Does CenteringPregnancy group prenatal care affect the birth experience of underserved women? a mixed methods analysis. J Immigr Minor Health 2017:19:415-22.
40 Chae SY, Chae MH, Kandula S, et al. Promoting improved social support and quality of life with the CenteringPregnancy® group model of prenatal care. Arch Womens Ment Health 2017;20:209-20.

41 Cox R, Obichere R, Knoll F. A study to compare productivity and cost of the CenteringPregnancy model of prenatal care with a traditional prenatal care model. Final report to March of Dimes 2006.

42 Catling CJ, Medley N, Foureur M, et al. Group versus conventional antenatal care for women. Cochrane Database Syst Rev 2015;4:CD007622.

43 Byerley BM, Haas DM. A systematic overview of the literature regarding group prenatal care for high-risk pregnant women. BMC Pregnancy Childbirth 2017;17:329.

44 Carter EB, Barbier K, Sarabia R, et al. Group versus traditional prenatal care in low-risk women delivering at term: a retrospective cohort study. J Perinatol 2017;37:769-71.

45 Abshire C, Mcdowell M, Crockett $\mathrm{AH}$, et al. The impact of CenteringPregnancy group prenatal care on birth outcomes in Medicaid eligible women. J Womens Health 2019;28:919-28.

46 Cunningham SD, Lewis JB, Shebl FM, et al. Group prenatal care reduces risk of preterm birth and low birth weight: a matched cohort study. J Womens Health 2019;28:17-22.

47 Australian Government Department of Health. Medicare Canberra, Australia, 2020. Available: www.health.gov.au/health-topics/medicare

48 Australian Institute Health and Welfare. Australia's mothers and babies 2018: in brief. Perinatal statistics series no. 36. Cat. no. PER 108. Canberra: AlHW, 2020.

49 Yelland J, Riggs E, Szwarc J, et al. Bridging the gap: using an interrupted time series design to evaluate systems reform addressing refugee maternal and child health inequalities. Implement Sci 2015;10:62.

50 Group Pregnancy Care Study. Group pregnancy care, 2021. Available: https://www.strongerfutures.org.au/group-pregnancy-care

51 Victorian Foundation for Survivors of Torture. Rebuilding shattered lives. Melbourne: Victorian Foundation for Survivors of Torture, 1998.

52 Mitchell P, Pirkis J, Hall J, et al. Partnerships for knowledge exchange in health services research, policy and practice. J Health Serv Res Policy 2009;14:104-11.

53 Victorian Foundation for Survivors of Torture. Professional development: training Melbourne, 2020. Available: https:// foundationhouse.org.au/learn-with-us/

54 Centre G. Workplace training, 2020. Available: https://groupwork. com.au

55 Horton T, Illingworth J WW. The spread challenge: how to support the successful uptake of innovations and improvements in health care. London: health Foundation, 2018.

56 Greenhalgh T, Wherton J, Papoutsi C, et al. Beyond adoption: a new framework for theorizing and evaluating Nonadoption, abandonment, and challenges to the scale-up, spread, and sustainability of health and care technologies. J Med Internet Res 2017;19:e367.

57 Greenhalgh T, Papoutsi C. Spreading and scaling up innovation and improvement. BMJ 2019;365:I2068.

58 Greenhalgh T, Papoutsi C. Studying complexity in health services research: desperately seeking an overdue paradigm shift. BMC Med 2018;16:95.

59 Liu J, Davidson E, Bhopal R, et al. Adapting health promotion interventions to meet the needs of ethnic minority groups: mixedmethods evidence synthesis. Health Technol Assess 2012;16:1-469.

60 Tannahill C, Sridharan S. Getting real about policy and practice needs: evaluation as a bridge between the problem and solution space. Eval Program Plann 2013;36:157-64.

61 Curtis $E$, Jones $R$, Tipene-Leach $D$, et al. Why cultural safety rather than cultural competency is required to achieve health equity: a literature review and recommended definition. Int $J$ Equity Health 2019;18:174.

62 Ellis BH, Kia-Keating M, Yusuf SA, et al. Ethical research in refugee communities and the use of community participatory methods. Transcult Psychiatry 2007;44:459-81.

63 Riggs E, Yelland J, Szwarc J, et al. Promoting the inclusion of Afghan women and men in research: reflections from research and community partners involved in implementing a 'proof of concept' project. Int J Equity Health 2015;14:13.

64 Harris PA, Taylor R, Minor BL, et al. The REDCap Consortium: building an international community of software platform partners. $J$ Biomed Inform 2019;95:103208.

65 StataCorp. Stata statistical software: release 16. College Station, Texas: StatCorp LP, 2020.

66 Penfold RB, Zhang F. Use of interrupted time series analysis in evaluating health care quality improvements. Acad Pediatr 2013;13:S38-44. 
67 Lopez Bernal J, Cummins S, Gasparrini A. The use of controls in interrupted time series studies of public health interventions. Int $J$ Epidemiol 2018;47:2082-93.

68 Langley G, Moen R, Nolan K. The improvement guide: a practical approach to enhancing organizational performance. San Francisco CA: Jossey-Bass, 2009.

69 Bisognano M, Cherouny PH, Gullo S. Applying a science-based method to improve perinatal care: the Institute for healthcare improvement perinatal improvement community. Obstet Gynecol 2014:124:810-4.

70 Yelland J, Biro MA, Dawson W, et al. Bridging the language gap: a co-designed quality improvement project to engage professional interpreters for women during labour. Aust. Health Review 2017;41:499-504.

71 Green J, Willis K, Hughes E, et al. Generating best evidence from qualitative research: the role of data analysis. Aust $N Z \mathrm{~J}$ Public Health 2007;31:545-50.

72 Small R, Yelland J, Lumley J, et al. Cross-Cultural research: trying to do it better. 2. enhancing data quality. Aust N Z J Public Health 1999;23:390-5.

73 Mollica RF, Caspi-Yavin Y, Bollini P, et al. The Harvard trauma questionnaire. J Nerv Ment Dis 1992;180:111-6.

74 Cox JL, Holden JM, Sagovsky R. Detection of postnatal depression. development of the 10-item Edinburgh postnatal depression scale. Br J Psychiatry 1987;150:782-6.
75 Small R, Lumley J, Yelland J, et al. The performance of the Edinburgh postnatal depression scale in English speaking and non-English speaking populations in Australia. Soc Psychiatry Psychiatr Epidemiol 2007;42:70-8.

76 Hegarty K, Fracgp BR, Bush R, et al. The composite abuse scale: further development and assessment of reliability and validity of a multidimensional partner abuse measure in clinical settings. Violence Vict 2005;20:529-47.

77 QSR International. NVivo 12 plus. version 12. Doncaster Victoria, Australia: QSR International Pty Ltd, 2018.

78 Drummond M, Sculpher M, Torrance G. Methods for the economic evaluation of health care programmes. 3rd ed. UK: Oxford University Press, 2005.

79 National Health and Medical Research Council. Ethical conduct in research with Aboriginal and Torres Strait Islander peoples and communities: guidelines for researchers and stakeholders. Canberra: Commonwealth of Australia, 2018.

80 Block K, Riggs E, Haslam NJV. Ethics in Research with Refugees and Asylum Seekers: Processes, Power and Politics. In: Block K, Riggs E, Haslam NJV, eds. Values and vulnerabilities: the ethics of research with refugees and asylum seekers. Toowong, Qld: Australian Academic Press, 2013: 3-19.

81 Mackenzie C, McDowell C, Pittaway E. Beyond 'Do No Harm': the challenge of constructing ethical relationships in refugee research. $J$ Refug Stud 2007;20:299-319. 